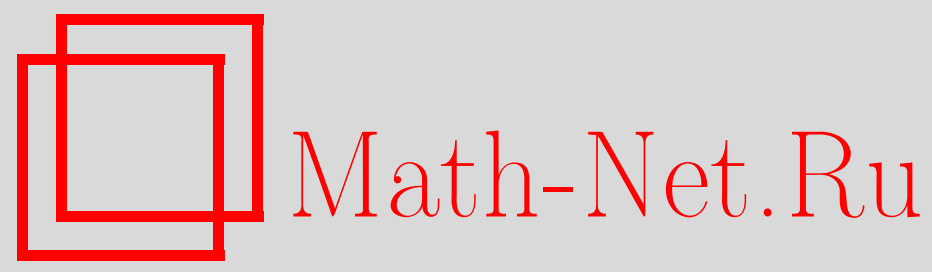

Р. Ф. Хабибуллин, Асимптотический ряд для многочленов Бесселя, Матем. заметки, 2005, том 77, выпуск 6, 948-950

DOI: https://doi.org/10.4213/mzm2549

Использование Общероссийского математического портала Math-Net.Ru подразумевает, что вы прочитали и согласны с пользовательским соглашением http://www.mathnet.ru/rus/agreement

Параметры загрузки:

IP: 54.174 .149 .18

26 апреля 2023 г., 15:33:20

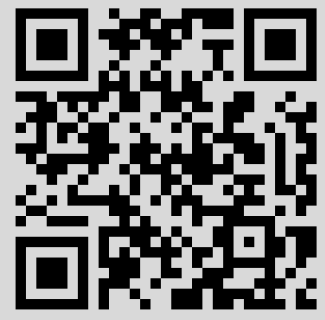




\section{АСИМПТОТИЧЕСКИЙ РЯД ДЛЯ МНОГОЧЛЕНОВ БЕССЕЛЯ}

\section{Р.Ф. Хабибуллин}

1. Постановка задачи. Многочлены Бесселя $\boldsymbol{B}_{n}(z), \operatorname{deg} \boldsymbol{B}_{n} \leqslant n-1, \boldsymbol{B}_{n} \not \equiv 0$, определяются как многочлены, ортогональные относительно комплексной весовой функции $\exp [1 / z]$, на (произвольой) замкнутой, жордановой, спрямляемой кривой $F$, содержащей внутри точку 0 , т.е.

$$
\int_{F} B_{n}(z) z^{k} e^{1 / z} d z=0, \quad k=0, \ldots, n-1 .
$$

Здесь мы будем рассматривать "сжатые" многочлены: $B_{n}^{*}(z):=B_{n}(z /(a n))$, где $a \in \mathbb{R}, a \neq 0$. Многочлены $B_{n}^{*}$ ортогональны относительно "переменного" (т.е. зависящего от номера многочлена) веса:

$$
\int_{F} B_{n}^{*}(z) z^{k} e^{a n / z} d z=0, \quad k=0, \ldots, n-1 .
$$

Асимптотика (при $n \rightarrow \infty$ ) многочленов, ортогональных относительно комплексного переменного веса, изучалась Гончаром, Рахмановым [1] и Аптекаревым [2] в связи с задачами о скорости аппроксимации аналитических функций рациональными. В частном случае сжатых многочленов Бесселя, общие теоремы работ [1], [2] могут быть детализированы и существенно уточнены. В настоящей заметке приводится теорема о полном асимптотическом ряде по степеням $1 / n$ для многочленов Бесселя $B_{n}^{*}$.

2. Равновесная мера. 1. Важную роль в общих асимптотических теоремах работ [1] и [2] играет (единственная) экстремальная мера $\lambda$, обеспечивающая равновесие логарифмического потенциала

$$
V_{\lambda}(z):=\int_{F} \ln \frac{1}{|t-z|} d \lambda(t)
$$

во внешнем поле $f \in \operatorname{Harm}(\Omega), F \Subset \Omega$ :

$$
\begin{aligned}
& V_{\lambda}+f=\gamma_{\Delta} \text { на } \Delta:=\operatorname{Supp}(\lambda) \subseteq F, \\
& V_{\lambda}+f \geqslant \gamma_{\Delta} \text { на } F .
\end{aligned}
$$

Если гладкая кривая $F$ и равновесная мера $\lambda$ удовлетворяют условию “симметрии":

$$
\frac{\partial\left(V_{\lambda}+f\right)}{\partial n_{+}}=\frac{\partial\left(V_{\lambda}+f\right)}{\partial n_{-}} \quad \text { на } \Delta,
$$

то говорят, что пара $(F, f)$ обладает $S$-свойством; этот факт будем обозначать $(F, f) \in S$.

2 . В случае внешнего поля $f(z)=\operatorname{Re}(-a /(2 z))$, соответствующего соотношениям ортогональности (1), задача равновесия (2) при условии (3) может быть решена в явном виде. Это можно сделать воспользовавшись интегральньм представлением для сжатых многочленов:

$$
B_{n}^{*}(z)=e^{-a n / z} \frac{1}{2 \pi i} \int_{\delta} \frac{\zeta^{2 n} e^{a n / \zeta} d \zeta}{(\zeta-z)^{n+1}}
$$

где контур $\delta$ - круговой контур вокруг точки $z$, ориентированный так же, как и $F$. Для простоты записи будем рассматривать здесь значение параметра $a$, равное 2 . Из формулы (4) можно заключить, что комплексный потенциал равновесной меры имеет вид

$$
\mathscr{V}_{\lambda}(z)=\frac{1}{z}+\frac{\sqrt{z^{2}+1}}{z}-1-2 \ln \left(z+1+\sqrt{z^{2}+1}\right)+\ln \left(1+\sqrt{z^{2}+1}\right),
$$

Работа выполнена при финансовой поддержке фонда INTAS, грант № 03-516637, и программы "Ведущие научные школы", грант № НШ-1551.2003.1. 
носитель $\Delta$ равновесной меры $\lambda$ является дугой (с концами в точках $-i, i$ ) трансцендентной кривой, задаваемой уравнением

$$
\log \left|x+\sqrt{x^{2}+1}\right|=\operatorname{Re} \frac{\sqrt{x^{2}+1}}{x}
$$

а производная $\lambda^{\prime}=d \lambda / d x$ этой меры равна

$$
\lambda^{\prime}(x)=\frac{1}{\pi i} \frac{\sqrt{x^{2}+1}}{x^{2}} .
$$

Из (5) вытекает следующее соотношение для комплексного потенциала на дуге $\Delta$ :

$$
\left(\mathscr{V}_{\lambda}\right)_{+}(x)+\left(\mathscr{V}_{\lambda}\right)_{-}(x)=\frac{2}{x}+2 \Gamma, \quad x \in \Delta,
$$

где

$$
\Gamma=-1-\ln (2 x)+\ln (-x), \quad x \in \Delta .
$$

Подставив в последнюю формулу значение $x=-i$, получим, что так называемая константа равновесия $\gamma_{\Delta}=\operatorname{Re} \Gamma$ равна

$$
\gamma_{\Delta}=-1-\ln 2 \text {. }
$$

Как известно [1], [2], главный член асимптотики вне носителя $\Delta$ равновесной меры $\lambda$ многочленов, ортогональных относительно комплексного переменного веса, выражается следующей функцией:

$$
\varphi_{n}(z)=\exp \left(\Gamma-\mathscr{V}_{\lambda}(z)\right)=\frac{(-1)^{n}}{2^{n}} \frac{\left(z+1+\sqrt{z^{2}+1}\right)^{2 n}}{\left(1+\sqrt{z^{2}+1}\right)^{n}} \exp \left(-n \frac{1+\sqrt{z^{2}+1}}{z}\right) .
$$

При $z \rightarrow \infty$ имеем $\varphi_{n}(z) \sim(-1)^{n}(2 / e)^{n} z^{n}$; тем самым, $C_{n} \varphi_{n}(z) \sim z^{n}$, где мы положили

$$
C_{n}:=(-1)^{n}\left(\frac{e}{2}\right)^{n}
$$

3. Основной результат. Основньм результатом настоящей работы является следующая теорема, в которой дается полное асимптотическое (при $n \rightarrow \infty$ ) разложение для полиномов $B_{n}^{*}$ вне дуги $\Delta$ носителя равновесной меры $\lambda$.

Теорема 1. Пусть $B_{n}^{*}(z)$ - последовательность сжатых полиномов Бесселя с единичным старшим коэффичиентом, функиии $\varphi_{n}$ определены в (6), а константы $C_{n}-\boldsymbol{\theta}(7)$. Тогда для $z \in \mathbb{C} \backslash \Delta$ отношение $B_{n}^{*}(z) /\left(C_{n} \varphi_{n}(z)\right)$ имеет следуюшее асимптотическое разложение по степеням $1 / n$ :

$$
\frac{B_{n}^{*}(z)}{C_{n} \varphi_{n}(z)} \sim \frac{1}{2}\left(\beta(z)+\beta^{-1}(z)\right)\left[1+\sum_{k=1}^{\infty} \frac{\Pi_{k}(z)}{n^{k}}\right] \quad \text { npu } \quad n \rightarrow \infty,
$$

где $\beta=((z-i) /(z+i))^{1 / 4}$. Разложсение (8) справедливо равномерно на компактах в $\overline{\mathbb{C}} \backslash \Delta$. функиии $\Pi_{k}$ голоморфны в $\mathbb{C} \backslash \Delta$ и могут быть найдены явно.

Коэффициент при $1 / n$ в разложении (8) имеет следующий вид:

$$
\Pi_{1}(z)=\frac{\pi i z\left(3 z^{2}-7\right)}{24\left(z^{2}+1\right)^{2}}+\left(z-\sqrt{z^{2}+1}\right) \frac{5 \pi i z^{2}}{12\left(z^{2}+1\right)^{2}} .
$$

Подставляя (6), (7) и (9) в (8), получаем, что для многочленов Бесселя $B_{n}^{*}$ равномерно на компактах из $\mathbb{C} \backslash \Delta$ справедливо следующее асимптотическое представление при $n \rightarrow \infty$ :

$$
\begin{aligned}
B_{n}^{*}(z)= & e^{n} \frac{\left(z+1+\sqrt{z^{2}+1}\right)^{2 n}}{\left(1+\sqrt{z^{2}+1}\right)^{n}} \exp \left(-n \frac{1+\sqrt{z^{2}+1}}{z}\right)\left(\frac{\sqrt{z-i}+\sqrt{z+i}}{2\left(z^{2}+1\right)^{1 / 4}}\right) \\
& \times\left(1+\left[\frac{\pi i z\left(3 z^{2}-7\right)}{24\left(z^{2}+1\right)^{2}}+\left(z-\sqrt{z^{2}+1}\right) \frac{5 \pi i z^{2}}{12\left(z^{2}+1\right)^{2}}\right] \frac{1}{n}+O\left(\frac{1}{n^{2}}\right)\right) .
\end{aligned}
$$


4. Заключение. Теорема 1 была получена методом, основанньм на исследовании некоторой матричной задачи Римана, предложенной Фокасом, Итсом и Китаевым (см. [3]). Асимптотика решения этой матричной задачи ищется методом перевала для матричных задач Римана, предложенньп и разработанным Дейфтом и соавторами (см., например, [4]-[7]). Этот метод получил в последнее время широкое применение при исследовании асимптотических свойств ортогональьх полиномов. Среди работ в этом направлении отметим [8], где были получены асимптотические представ ления для полиномов, ортогональных на вещественной оси относительно вещественнозначного переменного веса вида $h_{n}(x)=e^{-2 n Q(x)}, Q$ - фиксированньй многочлен, а также [9], где были исследованы обобщенные полиномы Якоби.

Автор выражает признательность А. И. Аптекареву за полезные советы и помощь в подготовке этой работы.

\section{СПИСОК ЦИТИРОВАННОЙ ЛИТЕРАТУРЫ}

1. Гончар А. А., Рахманов Е. А. // Матем. сб. 1987. Т. 134. №3. С. 306-352. 2. Аптекарев А. И. // Матем. сб. 2002. Т. 193. №1. С. 3-72. 3. Fokas A., Its A., Kitaev A. // Comm. Math. Phys. 1992. V. 147. P. 395-430. 4. Deift P. Orthogonal Polynomials and Random Matrices: A Riemann-Hilbert Approach. Reprint of the 1998 original. Providence, RI: Amer. Math. Soc., 2000. 5. Deift P. et al. // Intern. Math. Res. Notes. 1997. V. 16. P. 759-782. 6. Deift P. et al. // Comm. Pure Appl. Math. 1999. V. 52. №11. P. 1335-1425. 7. Deift P. et al. // Comm. Pure Appl. Math. 1999. V. 52. P. 1491-1552. 8. Ercolani N. M., McLaughlin K. D. T.-R. Asymptotics of the partition function for random matrices via Riemann-Hilbert techniques, and application to graphical enumeration // IMRM no. 14. 2003. 9. Vanlessen M. The Riemann-Hilbert Approach to Obtain Strong Asymptotics for Orthogonal Polynomials and Universality in Random Matrix Theory. Ph.D. thesis. Leuven, 2003.

Институт прикладной математики им. М. В. Келдьша РАН

Поступило E-mail: robson@rbcmail.ru 